\title{
Machine Learning-Based Adaptive Wireless Interval Training Guidance System
}

\author{
Myung-kyung Suh • Ani Nahapetian • \\ Jonathan Woodbridge • Mahsan Rofouei • \\ Majid Sarrafzadeh
}

Published online: 30 June 2011

(C) Springer Science+Business Media, LLC 2011

\begin{abstract}
Interval training has been shown to improve the physical and psychological performance of users, in terms of fatigue level, cardiovascular build-up, hemoglobin concentration, and self-esteem. Despite the benefits, there is no known automated method for formulating and tailoring an optimized interval training protocol for a specific individual that maximizes the amount of calories burned while limiting fatigue. Additionally, an application that provides the aforementioned optimal training protocol must also provide motivation for repetitious and tedious exercises necessary to improve a patient's adherence. This paper presents a system that efficiently formulates an optimized interval training method
\end{abstract}

This publication was partially supported by Grant Number T15 LM07356 from the NIH/National Library of Medicine Medical Informatics Training Program

M.-k. Suh $(\bowtie) \cdot J$. Woodbridge $\cdot$ M. Rofouei $\cdot$ M. Sarrafzadeh Computer Science Department, University of California,

Los Angeles, CA, USA

e-mail:dmksuh@cs.ucla.edu

J. Woodbridge

e-mail: jwoodbri@cs.ucla.edu

M. Rofouei

e-mail: mahsan@cs.ucla.edu

M. Sarrafzadeh

e-mail: majid@cs.ucla.edu

A. Nahapetian

Computer Science Department, California State University,

Northridge (CSUN),

Los Angeles, CA, USA

e-mail: ani@cs.ucla.edu

\section{A. Nahapetian}

Computer Science Department, UCLA,

Los Angeles, CA, USA

M. Sarrafzadeh

Wireless Health Institute, University of California,

Los Angeles, CA, USA for each individual by using data mining schemes on attributes, conditions, and data gathered from individuals exercise sessions. This system uses accelerometers embedded within iPhones, a Bluetooth pulse oximeter, and the Weka data mining tool to formulate optimized interval training protocols and has been shown to increase the amount of calories burned by $29.54 \%$ as compared to the modified Tabata interval training protocol. We also developed a behavioral cueing system that uses music and performance feedback to provide motivation during interval training exercise sessions. By measuring a user's performance through sensor readings, we are able to play songs that match the user's workout plan. A hybrid collaborative, content, and context-aware filtering algorithm incorporates the user's music preferences and the exercise speed to enhance performance.

Keywords wireless health · embedded system · exercise guidance system · interval training $\cdot$ wearable wireless sensors · music recommendation - social networks . rehabilitation $\cdot$ data mining $\cdot$ Bayesian-network $\cdot \mathrm{J} 48$ tree

\section{Introduction}

Interval training is a training protocol that requires participants to increase and decrease the workout intensity between exercise sessions and recovery periods. Interval training is a well-known exercise protocol that has been shown to strengthen and improve cardiovascular health [14]. Moreover, it has been shown to help with weight loss, rehabilitation, general fitness, and the reduction of heart and pulmonary diseases. It utilizes the body's energy production system by activating both aerobic and anaerobic energy sources. Dimeo [5] shows that among the group participating in interval training, complaints of fatigue did not increase and psychological distress (such as obsession, fear, interpersonal sensitivity, and phobic anxiety) were diminished. 
Tabata interval training developed by Izumi Tabata at the National Institute of Fitness and Sports in Tokyo, Japan is one example of an interval training protocol (Fig. 1). The Tabata protocol produced a $14 \%$ increase in the maximum aerobic capacity, and a $28 \%$ improvement in anaerobic capacity when taking short rest intervals between high intensity exercises [6].

Interval training provides many health benefits; however, properly following a given interval training protocol is not simple. Without equipment, such as a programmed treadmill, it is difficult to figure out one's exercise speed to follow a customized exercise protocol. As such, the usual interval training methods suffer from the limitations that the individual subject may not be able to monitor the current and future level of effort. As a result, the individual may not be able to follow an optimal training plan. Moreover, a challenge exists in establishing the proper level of intensity for each exercise cycle. If the intensity is insufficiently low for an exercise session plan, then the desired stress level is also insufficiently low and the outcome benefits degrade. On the other hand, if the exercise design prescribes a level of intensity that is excessive, then the subject will either suffer from the ill effects of excessive exertion or may be unable to complete the prescribed interval training. In addition, by monitoring one's heart rate during exercise, we can avoid exceeding a level where the heart accumulates blood lactate causing muscles to ache and fatigue.

Consider an interval training protocol that requires a 5 min walk at $3.5 \mathrm{MPH}$ followed by a 1 min walk at 4.2 $\mathrm{MPH}$ and is repeated several times with a maximum heart rate value less than 140. This model is extremely difficult to follow without the proper fitness equipment that provides a customized and optimal exercise protocol with heart monitoring sensors. This is in addition to the space and cost restrictions of traditional fitness equipment. Typical treadmills with interval training functions cost more than 1,000 dollars and have a belt size of 1,200 square inches or larger. In other words, most current interval training systems are expensive and restricted to gyms because of their size. Individuals without motivation can

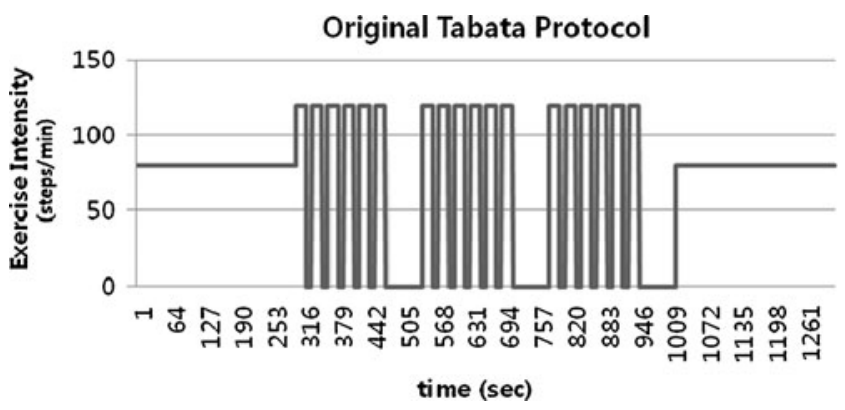

Fig. 1 Tabata interval training protocol. Intensity 120: 1 step per $0.5 \mathrm{~s}$. Intensity 75 : 1 step per $0.75 \mathrm{~s}$ be discouraged from following an interval training protocol. Even though some commercial iPhone interval training applications such as Interval Training GO [7] and iWorkout Muse [8] utilizes music for interval training, they do not monitor heart rate and not provide proper motivation such as proper music, game or social networks. Thus, it is important to design a customized interval training guidance system that is inexpensive, space efficient, and provides strong motivation for exercise.

In this paper, we present the following key contributions. We present our behavioral cueing system developed for the popular iPhone platform which costs less than 200 dollars and is less than 11 square inches to provide motivation and guidance in interval training exercise sessions. User data attained through GUI entry and sensor data is used by machine learning algorithms to provide an exercise schedule and music recommendations. Based on the obtained data, the developed system provides real-time feedback for music recommendations, scheduling, and social networks (Fig. 2). The developed system also gives sound, vibration, and image commands to help users follow a determined exercise schedule. Additionally, the system uses music, sensor readings, and social networking to encourage and motivate users to follow a healthy exercise plan.

To make a customized exercise schedule for an individual, the system collects data from the user, and generates a J48 decision tree. Based on the constructed decision tree, the system recommends conditions allowing the user to maximize the amount of exercise within the given heart rate range while avoiding fatigue. The system uses a Bayesian network method with content-based, collaborative, and context-aware recommendation techniques to recommend proper music to the user while exercising. In other words, by analyzing a user, user group, and the exercise context, our system recommends suitable songs for interval training with the Bayesian network structure. Also, based on the general characteristics of people, competitive group exercise methods incorporated into a game-like system motivate users to participate in interval training type exercises. By using social networks, such as sending emails to friends, uploading rankings on a shared website, etc., users can be effectively motivated.

Compared with the uncontrolled condition, the experiment shows that exercise commands help users to improve exercise accuracy by $89 \%$. Also, we obtained a $30 \%$ increase in the amount of calories burned compared with the original Tabata interval training protocol. Additionally, signs of improvement in the amount of the calorie expenditure appeared within 2 weeks, since the decision tree and the interval training that originally helps cardiovascular build-up were combined together. Compared with the method that recommends music preferred by people who share the same age range, gender, similar exercise 


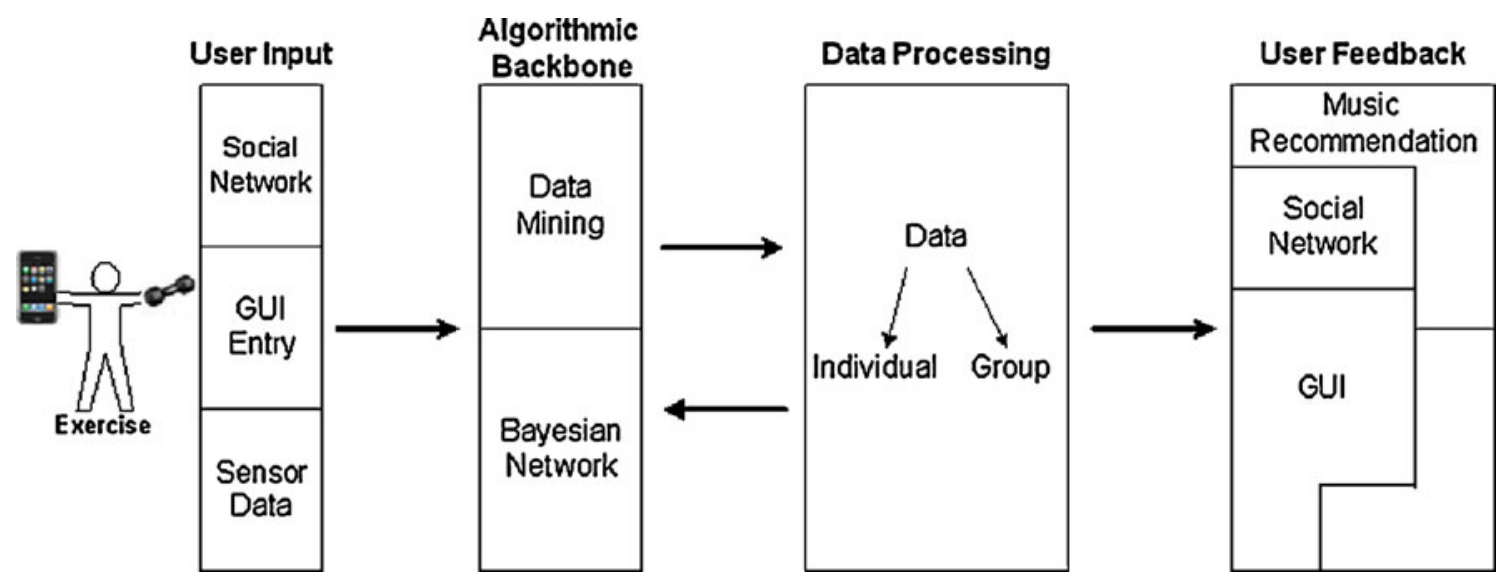

Fig. 2 Overall system structure

intensity and residential area, Bayesian networks-based recommendation method shows better results for selecting suitable exercise music while interval training

\section{Background and related works}

Recent advances in sensors, smart phones and wireless technology have made a new generation of health and fitness monitoring systems available. Previously, athletes and patients had to visit athletic centers or hospitals to monitor their health using large fitness monitoring systems. However, with recent advances in technology, people supplement (or even tailor) this process using sensors and handheld systems outside of the traditional institutions. Moreover, these systems have the power to give precise and real-time feedback, using data collected by sensors (such as accelerometers, pressure sensors, and gyroscopes). Since heart rate readings are highly related to the intensity of exercise, monitoring heart rate is helpful to avoid excessive intensity while exercising. Tapia [9] introduces a real-time activity and intensity recognition system, using five triaxial wireless wearable accelerometer sensors and a wireless heart rate monitor. The system transmits raw data in realtime and their fast decision tree classifiers efficiently compute activity types and intensities.

Terry [10] and Karageorghis [11] examine how the rhythm of music related to personal factors and situational factors promotes more exercise with less stress. Working out in time to music increases the likelihood of strenuous exercises for longer periods of time for users in a broad range of level of fitness. Listening to music provides benefits such as improvement in mood, pre-event activation or relaxation, dissociation from unpleasant feelings such as pain and fatigue, and reduced rating of perceived exertion (RPE), especially during aerobic training. A system that recommends music based on a user's preference, profile, and context is useful since downloading and choosing different music every time is inconvenient.

General recommendation systems use one of contentbased, collaborative, or context-aware techniques. A content-based filtering system selects items based on the correlation between the content of the items and the user's preferences, as opposed to a collaborative filtering system that chooses items based on the correlation among people with similar preferences. Collaborative filtering is the process of filtering for information or patterns using techniques involving collaboration among multiple agents who share similarities in attributes. Cano [13]presented Musicsurfer using the content-based music recommendation technique by extracting instrumentation, rhythm, and harmony from music signal using similarity metrics. The Genius feature in iTunes eight [14] automatically generates a playlist of songs from the user's library that are similar to the selected song, using the collaborative filtering technique. The LIBRA system [15] recommends books by using content-based and collaborative filtering. Many systems use hybrid features of content-based and collaborative filtering to recommend items. Those systems combine knowledge about users who liked a set of items with knowledge of a particular content feature associated with the item in one user's set. User context is any information that can be used to characterize the situation of an entity. Context includes location of use and the collection of nearby people, places, and accessible devices as well as changes to these nearby objects. It may include lighting, noise level, network connectivity, communication costs, communication bandwidth, time, proximity, activity, and even social situations [16]. A context-aware system provides a user with relevant information and services based on one's current context and filters recommended contents for the context [17]. Park [18] developed a context-aware music recommendation system using fuzzy Bayesian networks using user profile, sensor data such as 
temperature, humidity, noise, weather, etc., and internet data sources. The PILGRIM recommendation system [19] uses mobility-aware filtering techniques with a GPS system. CAMA [16] also applies a user's clicking behavior context to recommend websites. Van Setten [17] developed the mobile tourist application which takes into account the user's interests and contextual factors such as the place mostly recently visited.

Competitive group interval training is another form of user motivation, with known beneficial effects on physiological functioning. The experimental results (Kilpatrick [20], Table 1) indicate that sport participants are more motivated to engage in physical activity as a means of enjoyment than to achieve positive health benefits. Sports participation is strongly related to affiliation, competition, enjoyment, and challenge. A program of aerobic, and endurance activities such as interval training, undertaken in a group setting, stimulates and improves physiological and cognitive functions and subject wellbeing (Williams [21]). Since doing exercising together can help people maintain affiliation with friends and promote more exercise, systems related to social networks are helpful to provide motivations.

\section{System overview}

Without fitness equipment, interval training is very difficult to follow since individuals are unable to precisely determine their current exercise intensity. On the other hand, traditional fitness equipment has significant space and cost restrictions. Therefore, the use of an inexpensive mobile hand held device with embedded sensors can be an

Table 1 Ranking of exercise motivation: Range $=1$ (most important) to 14 (least important)

\begin{tabular}{ll}
\hline Subscale & Ranking \\
\hline Affiliation & 2 \\
Appearance & 12 \\
Challenge & 4 \\
Competition & 1 \\
Enjoyment & 3 \\
Health pressures & 14 \\
Ill-health avoidance & 13 \\
Nimbleness & 8 \\
Positive health & 7 \\
Revitalization & 5 \\
Social recognition & 9 \\
Strength and endurance & 6 \\
Stress management & 10 \\
Weight management & 11 \\
\hline
\end{tabular}

effective means for guiding interval training exercises. It should satisfy requirements such as ease of use, compatibility, and communicability that are key issues related to mobile device use and adoption by individuals [12].

\subsection{System components}

The developed system requires user data such as a GUI input (e.g. user's age, sex, email, desired exercise time, calorie expenditure, etc.), sensor data, and previous exercise history. Based on this data, the system designs a custom game-like interval training protocol that provides real-time feedback and an optimized schedule. As the user exercises, the data is accumulated in our web-database to reflect the user's history to the future exercise (Fig. 3).

To measure the accuracy of the exercise, we used an iPhone with a 3 -axis accelerometer and a $3 \mathrm{G}$ cellular connection for communicating with the web-database. Alive Technologies Bluetooth pulse oximeter was used in our experiment to monitor heart rates. Heart rate data is measured and sent at $0.1 \mathrm{~s}$ intervals. A C4.5 (J48, [22]) decision tree generated by the Weka data mining tool along with data mining techniques were used to construct a customized exercise schedule that maximizes calorie consumption.

\subsection{A developed iPhone application}

Sarker [23] mentions factors influencing the use of mobile hand held devices by individuals, including technology, communication/task characteristics, modalities of mobility, and context (Fig. 4). Those same features support our choice of a smart phone platform for our system.

Usability is extremely important to mobile technologies. An iPhone's 3.5 in. multi-touch display with 480-by-320pixel resolution allows users to navigate by touching the screen. The multi-touch display layers a protective shield over a capacitive panel that senses touches using electrical fields. It then transmits that information to the LCD screen below it, and iPhone's software enables the flick, tap, and pinch gestures. iPhone's user interface also supports multiple languages and characters for users worldwide.

Poor network characteristics act as severe inhibitors to use and adoption. For example, the lack of coverage in many areas tend to reduce the sense of freedom and safety in many subject's minds. The iPhone 3G uses a technology protocol called HSDPA (High-Speed Downlink Packet Access) to download data quickly over UMTS (Universal Mobile Telecommunications System) networks. Accessing the internet to download information is twice as fast on $3 \mathrm{G}$ networks as on $2 \mathrm{G}$ EDGE networks. Since the iPhone $3 \mathrm{G}$ meets worldwide standards for cellular communications, a user can make calls and surf the web from practically 
Fig. 3 An overall system design

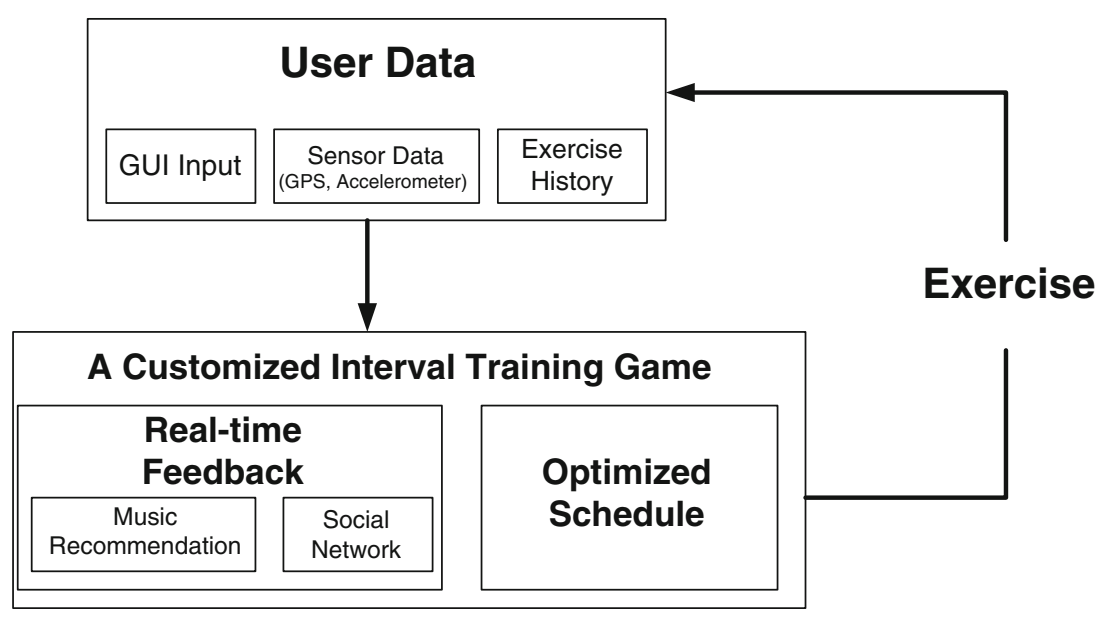

anywhere. When a user is not in a $3 \mathrm{G}$ network area, the iPhone uses a GSM network for calls and an EDGE network for data. Therefore, the iPhone's 3G internet connectivity, and light size can be a good source of communicability and modalities of mobility.

According to the market research group NPD, Apple's iPhone $3 \mathrm{G}$ topped the sales charts in the third quarter of 2008. Therefore, many iPhone users and developers share information regarding iPhone systems and applications via several websites or magazines. In addition, the number of interactions among iPhone user groups and developer groups continues to increase.

Additionally, research has shown that for a variety of reasons, humans are attracted to the outdoor environment [2426]. These investigations have shown subjects encountering images of natural outdoor environments subjects experience a variety of positive psychological, social, and physiological outcomes. Since an iPhone's weight is only $133 \mathrm{~g}$, it is easy to carry to a outside environment setting where interval training can be performed. Moreover, an iPhone embeds a 3- axis accelerometer making it possible to monitor one's exercise status and give real-time feedback in outdoor environments. Accelerometers are currently among the most widely studied wearable sensors for activity recognition. The accuracy of exercise and the calorie consumption can be calculated by analyzing data obtained from three different axes of an accelerometer. Therefore, providing various positive psychological, social, and physiological effects, using an iPhone helps users exercise in the outdoor environment.

Rhythm of music related to the user's preference and situational conditions affect exercise and stress level [10, $11,27]$. For example, the change in tempo helps prolong the exercise time to fatigue [28]. And the tempo speeds up in order to motivate the user to speed up by synchronizing one's steps with the music [29]. Thus, music is a good source of motivation during interval training especially when the exercise method is tedious and repetitious. The portability of iPhone allows users to exercise while listening to music via iTunes [14] and reduce the feelings of over-exertion (Fig. 5).
Fig. 4 Factors influencing mobile handheld device use and adoption [23]

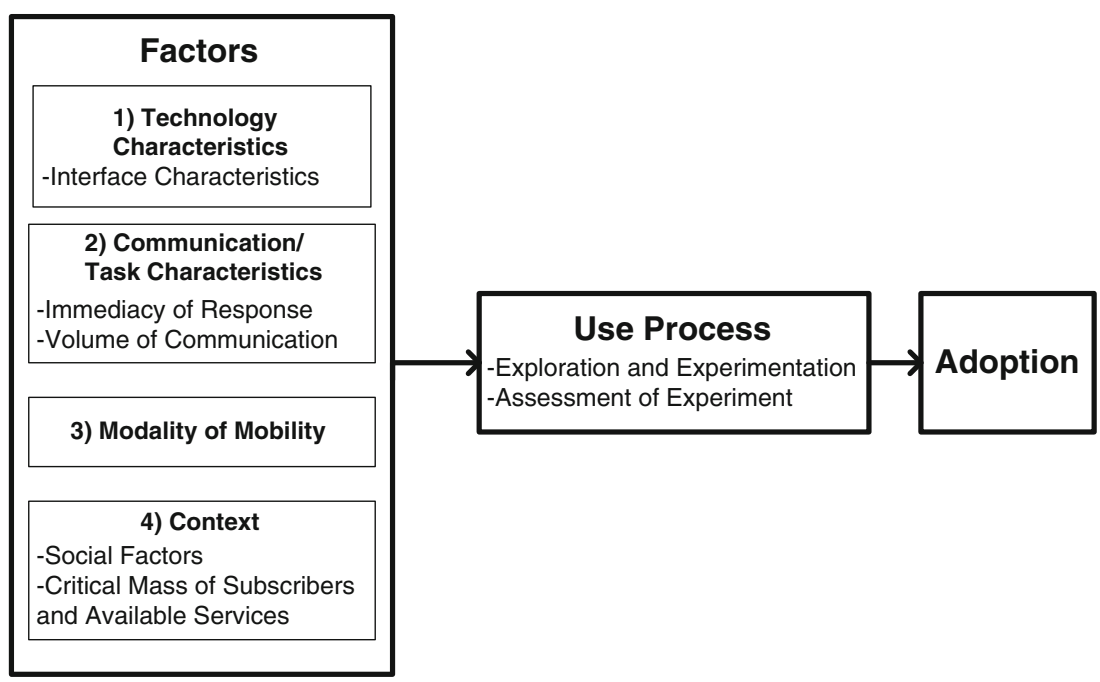




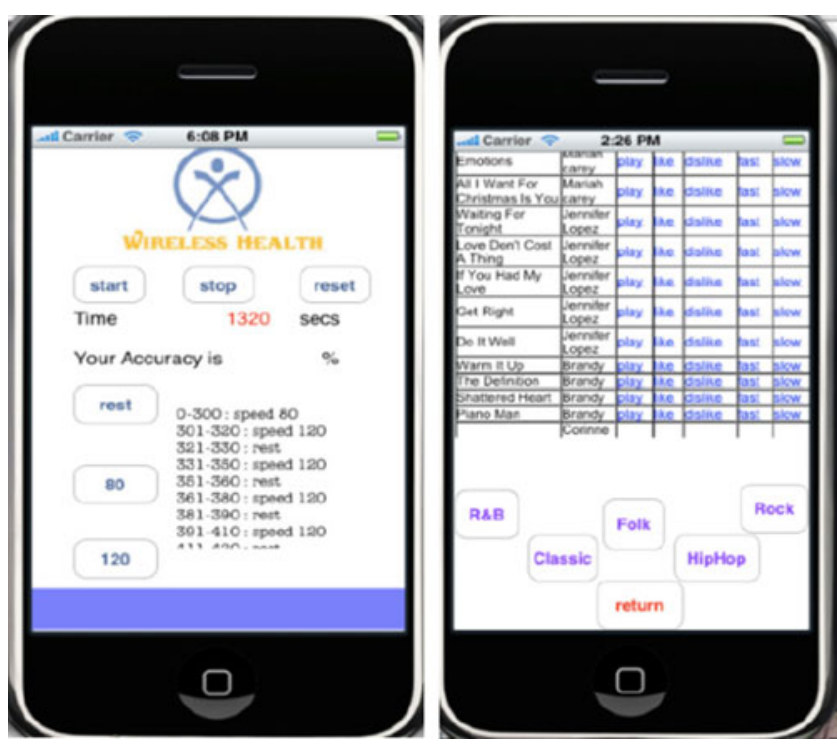

Fig. 5 A customized interval training system. This system uses accelerometer functionality, GPS, graphical, sound, vibration, etc. functionalities on an iPhone

\subsection{Social network application}

The user input screen requires user information including weight, height, the amount of time to exercise, and the amount of calories to be burned. With the inserted user input, the system design is customized to an interval training protocol. The following equation is used by the system to design a customized exercise plan for the user. With given user input values, the system provides a proper speed of exercise to burn the given amount of calories during the specified time. The system indicates the speed of exercises to the user with sounds, vibrations and images during the exercise session. When the user is unable to follow the schedule, the system dynamically changes the schedule to make a user to achieve his/her goals.

$\mathrm{W}=\mathrm{m} \cdot \mathrm{g} \cdot \mathrm{h} \cdot \mathrm{h} \_$rate $\cdot \mathrm{v}^{\prime} \cdot \mathrm{t}+\frac{1}{2} \cdot \mathrm{m} \cdot \mathrm{v} 2 \cdot \mathrm{t}(\mathrm{J})$

$\begin{array}{ll}\text { W } & \text { work }(\mathrm{J}, 1 \mathrm{~J}=0.24 \text { cal }) \\ \mathrm{m} & \text { mass }(\mathrm{kg}) \\ \mathrm{g} & \text { the gravitational constant }(\mathrm{kg} / \mathrm{m} 2) \\ \mathrm{V} & \text { the speed of running }(\mathrm{m} / \mathrm{s}) \\ \mathrm{v}^{\prime} & \text { the number of steps per second (steps/s) } \\ \mathrm{t} & \text { collapsed time }(\mathrm{s}) \\ \mathrm{h} & \text { height }(\mathrm{m}) \\ \mathrm{h} \text { rate } & \text { the rate of height lifted up when walking/running }\end{array}$

While exercising, our system provides three types of feedback consisting of sound, vibration and animation based on the developed schedule. For the animation command, it changes the color of the colored bar each time the person should step. For sound and vibration, the developed system gives either a short sound or vibration.

Accuracy $=\frac{\text { The number of correct steps }}{\text { The number of times commands are given }}$

The accuracy of the exercise is calculated by comparing the determined schedule of the interval training exercise with the exercise data collected via iPhone's 3-axis accelerometer. Activities are recognized by comparing the $\mathrm{x}, \mathrm{y}$ and $\mathrm{z}$-axis acceleration with threshold values. For instance, the accuracy of the exercise shown in Fig. 6 is $50 \%$, since only three steps among six given commands are completed. The system gives a lower score to the user who does not complete the interval training session accurately in order to motivate the user to increase the amount and preciseness of exercise in an effort to achieve a better score. In addition, a better score will result in more effective exercise regimen with more calories burned and motivation for winning the competition among friends group.

As mentioned in Section 2, Exercising in groups can help people maintain affiliation with friends and promote exercise. Our designed system encourages group workouts by incorporating social networks. By designing a social network-based interval training game, it is possible to encourage users to participate in interval training. The user's interactions with the iPhone are synchronized with the user's online profile registered in the web database of

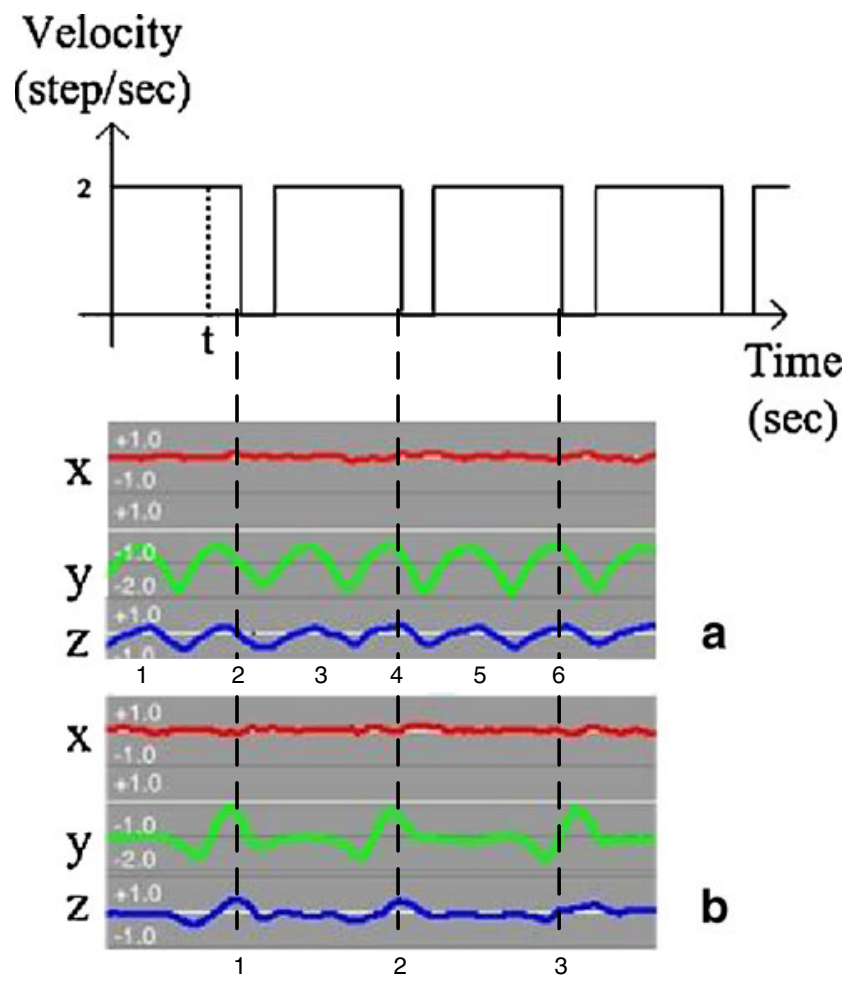

Fig. 6 Scheduled interval training and the accelerometer data for the accurate exercise (a) and the $50 \%$ accurate exercise (b) at time $t$ 
our system. These interactions are also registered to the user's friend group (a group of users who participate in the iPhone interval training guidance system together) in Fig. 7. E-mails containing the accuracy of the exercise sessions, exercise session time, the amount of calories burned, etc. can be sent to other members in the user's social networking group after an exercise session is completed. These emails increase the interaction among the user's friend group. Shared information increases the motivation to exercise by creating competition among friends. Our current work utilizes e-mails to provide a social network to users. However, combining the system with an existing social network application, such as Facebook [30], Twitter [31], etc., can further improve the experience.

\section{Approach}

\subsection{Protocol approach}

While exercising, the quantity of blood pumped by the heart increases to match the increased skeletal muscle demand. In addition, heart rate also acts as an indicator of exercise intensity. The more intense the activity, the faster your heart will beat. In contrast, immediately after stopping exercise activity, the heart rate should decrease [32]. Figure 8 indicates heart rate during 22 min modified Tabata interval training (Fig. 1). We can observe that the heart rate increased during exercise and decreased during rest.

Mizuo [33] denotes that rising heart rate curves have an exponential hyperbolic shape, and the falling heart rate curves are exponential when exercising.

HR of the Rising Curve $=\mathrm{Ae}^{-\beta \mathrm{t}} \sinh (\omega \mathrm{t})+\mathrm{C}$

HR of the Falling Curve $=A\left(1-e^{-\beta t}\right)+C$
As Borg [34] mentions, there is a relation between heart rate and lactic acid that causes fatigue. Therefore, by monitoring one's heart rate during exercise, we can increase the length of workouts by preventing a person's heart rate to exceed a fatiguing level. Also, if we decrease the amount of time for the heart rate to reach a certain level during rest, it will help increase the amount of exercise time within limited time. For example, if you can increase the time to reach a heart rate of $113 \mathrm{bpm}$ from 135 to $269 \mathrm{~s}$ in a $22 \mathrm{~min}$ period, you can exercise an additional 134 s (Fig. 9). Similarly, if you can reduce the amount of rest time, exercise time will be increased.

To maximize the amount of the calorie expenditure, the time constant of an exercise period should be maximized until the heart rate reaches a certain high level. In contrast, the time constant of a rest period that has an exponential curve characteristic should be minimized in order to reduce the time to reach a certain low level heart rate. As you can see in Fig. 9, if the initial exponential sine hyperbolic curve changes to the modified one that has a bigger time constant, the user can exercise longer and burn more calories until the heart rate value is less than or equal to 120 .

When observing the same heart rate curve from the same individual [35] several times, the curves are dramatically different. Thus it is hard to predict and guess future heart rate values with only the above equations. Instead, it is better to use a statistical and probabilistic approach to extract, compare and infer certain characteristics from the many different heart rate curves of an individual. By combining the information of the heart rate curve, such as the heart rate value and the time constant of the curve which characterizes the frequency response of the curve, along with other individual conditions, we can maximize the amount of calories burned by an individual during an interval training workout. Since each individual has his or her own unique conditions such as age, gender, and level of
Fig. 7 The method to get the friend group who compete with the user in our system

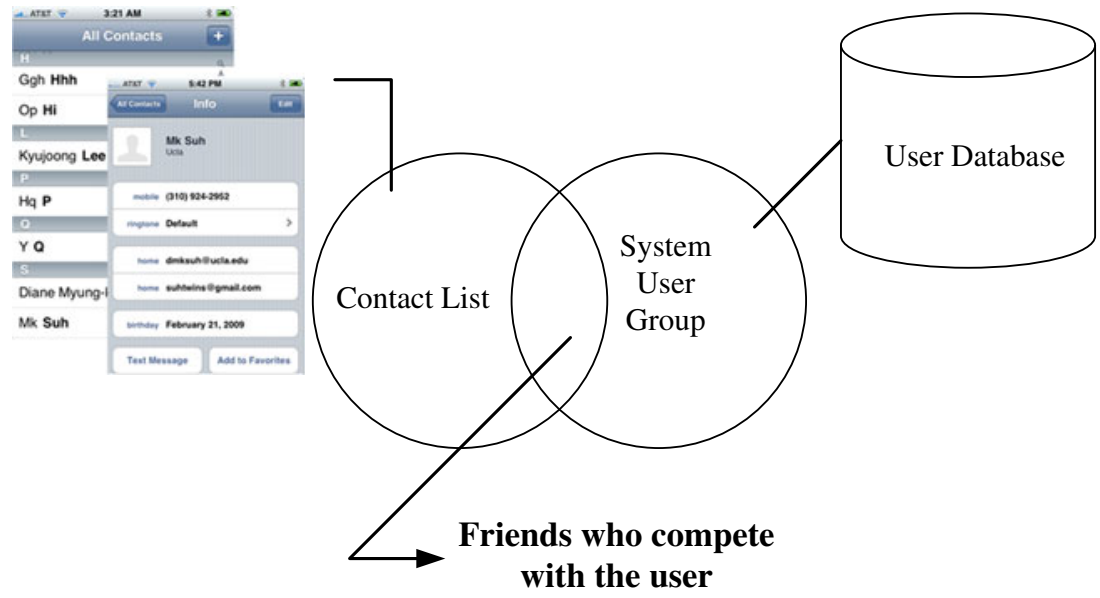




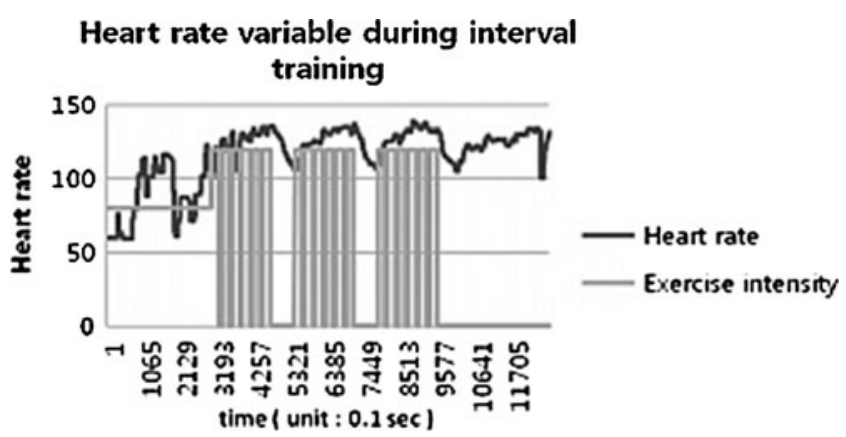

Fig. 8 Heart rate and exercise intensity during 22 min Tabata interval training

activity, which will in turn affect the heart rate values, we will use this information to help in the calculation of a user's optimal heart rate and fatigue level. Also, the regular use of pharmaceuticals by a person and the elapsed time after they are taken can drastically affect the heart rate curve [36-38]. Additionally, we need to take into account the amount of time after having a meal since this could also affect the heart rate, fatigue level, and other conditions related to exercise. In our experiments, we took into account all factors since these conditions can considerably affect an individual's heart rate.

\subsection{Music recommendation approach}

By using the nearest neighbor's information, the collaborative filtering technique generates a group of users who share a similar choice in music. The collaborative filtering technique used by our system reflects personal factors such as one's age, sex, cultural background, and education level that affect one's taste in music. A user's choice in music in the past can affect the list of recommended music by content-based filtering. The developed system recommends music that has something in common with music that the user preferred in the past. Thus, content-based recommendation systems with collaborative filtering analyze the

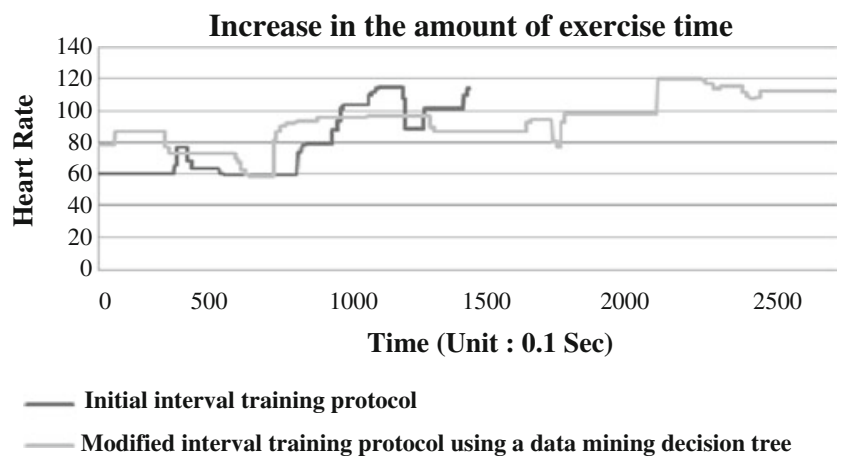

Fig. 9 Increase in the amount of time to reach heart rate 113 in 22 min Tabata interval training protocol content of the objects that a user has preferred in the past, and they recommend other relevant content by using one's history and the nearest neighbor's information [39-41]. Context aware technique helps recommendation system to choose items based on the user's current location, nearby people and objects, accessible devices, etc. Our system uses GPS data, accelerometer data, and user input to develop a context-aware pervasive system that reflects user's exercise context (Fig. 10).

Based on training data, a user model is created to enable the content-based filtering technique to classify unseen items. The training set consists of items found most interesting by the user. These items form training instances with inherent attributes such as tempo to a song. These attributes specify the class of the item based on either the rating of the user or on implicit evidence. The list of recommended items is determined with the class information. Our system classifies songs by its genre and tempo. The system uses these classifications to recommend songs to the user based on the user's history, background, exercise information, etc. The system classifies users based on age, gender, and residential location. Recommendations for users in the same group are affected by the annotations of other members of the group. In addition, the system keeps user's history, and recommends similar music that the user preferred as other filtering recommendation systems do. If there is music that only one specific individual does not like, this song will not be recommended for

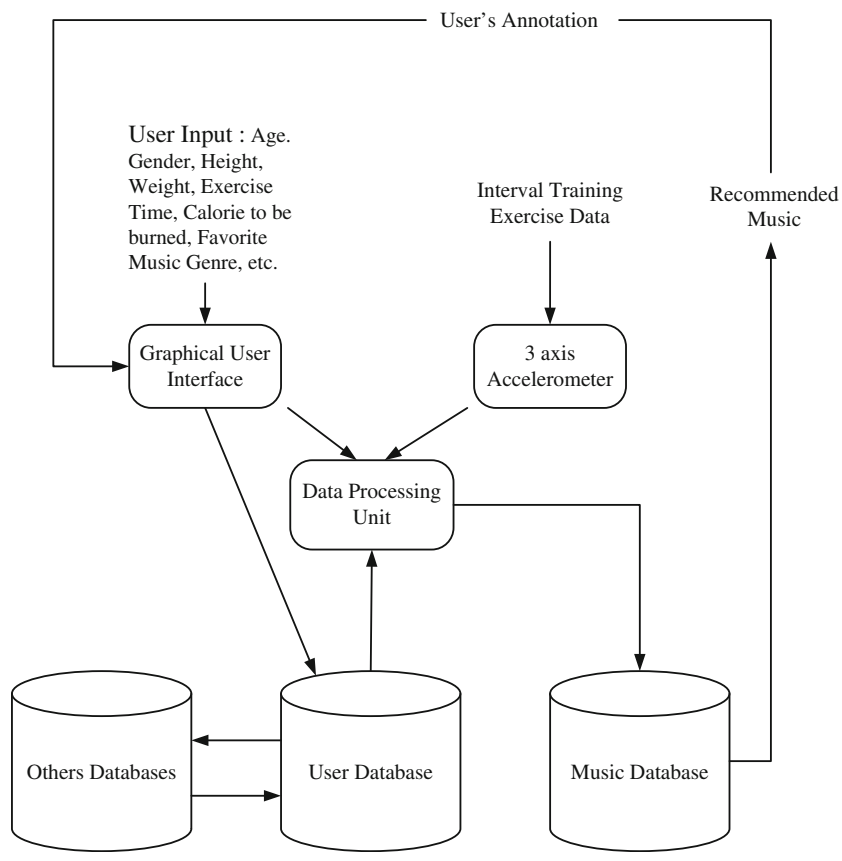

Fig. 10 The structure of context-aware, content-based, and collaborative music recommendation system 
that user, but still will be recommended to others until the rating is below the threshold level. Our system recognizes the exercise context by using user input such as exercise time, the amount of calories to be burned and sensor data stream from an accelerometer and GPS. Using this information, the system can recommend suitable music for the exercise context. Therefore, our system realizes the collaborative, content-based, and context-aware recommendation by analyzing the user's information, the group the user belongs to, the speed and the location of the exercise, etc. As user's annotation is accumulated in the database, the list of the recommended music is also updated and made more suitable for the user and the exercise context. Thus the recommended music is modified and adapted, as the number of annotation data increases.

Music preferences are determined by a listener's age, gender, ethnic group, residential area, family, peer group, etc. LeBlanc $[42,43]$ determined that the age of a music listener exerts a strong influence on overall preferences of music style and the speed of music. Christenson [44] denotes gender is central to the ways in which popular music is used and tastes are organized. Even though the underlying structure of music preference cannot be accounted for by reference to two or three factors, there are crucial difference between males and females in terms of their mapping of music types [45]. In addition, preferences in popular music also vary according to the neighborhood in which the music listener lives [46]. People can also be affected by their family and peers as well as their backgrounds (such as the listener's music training experience and level of education). There are additional factors that also affect the music preference shown in Fig. 11. Using the structure in Fig. 11 and information that can be obtained by the iPhone, such as sex, age, exercise intensity, and location data obtained by GPS embedded within an iPhone, etc., a sample Bayesian network structure in Fig. 12 is designed. Bayesian networks are a probabilistic model using a set of random variables and conditional relations. This probabilistic model attempts to make valid predictions based on only a sample of all possible observations. Peer groups are assumed to be formed by age, gender, and residential district since these factors affect perceptions of relationships [45]. The physiological enabling condition is assumed to be related to the exercise intensity information such as exercise time, and calories to be burned in the user's input. Based on the above assumptions, a Bayesian network model is obtained and is used to calculate the probability that the given song is recommended by people sharing similarities with the user. The probabilistic model would be quantified in the following way for the sample Bayesian network given in Fig. 12. The following

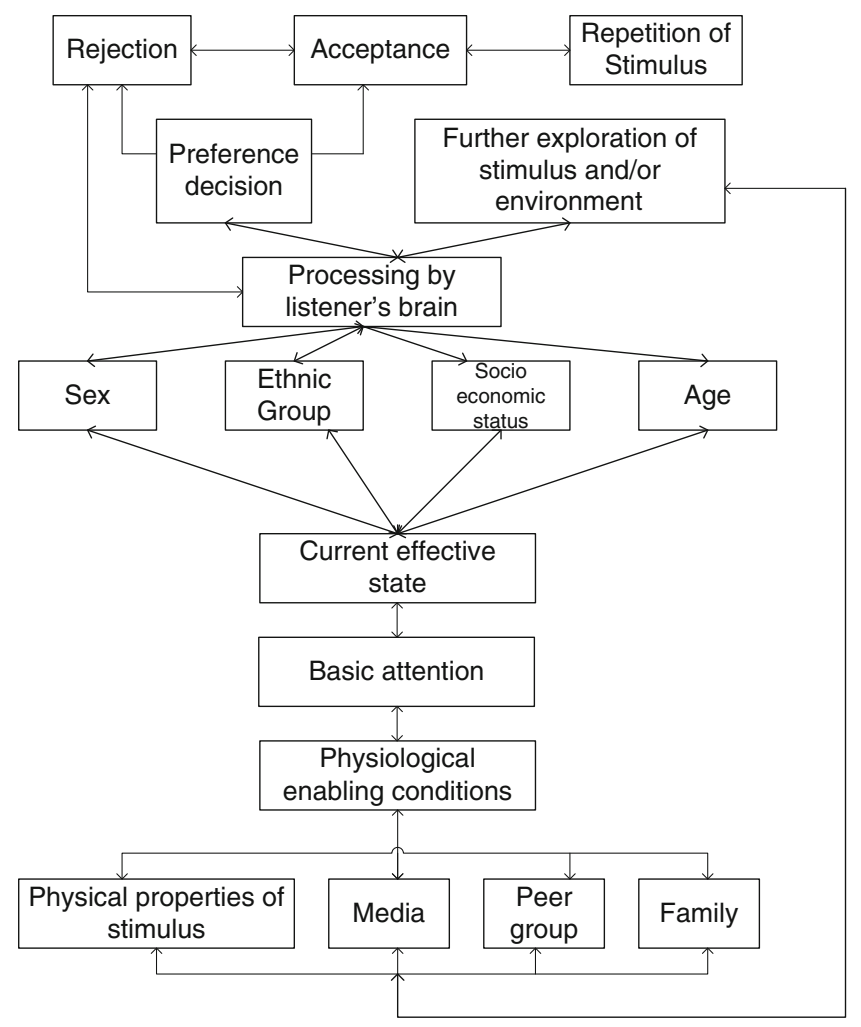

Fig. 11 Sources of variation in music preference

equation shows how to calculate the probability of recommending MusicA to a male user living in CityA between the ages of 10 and 19 years old and exercising at a speed of $0-9 \mathrm{ft} / \mathrm{s}$. When the obtained probability value is larger than the threshold value, the developed system

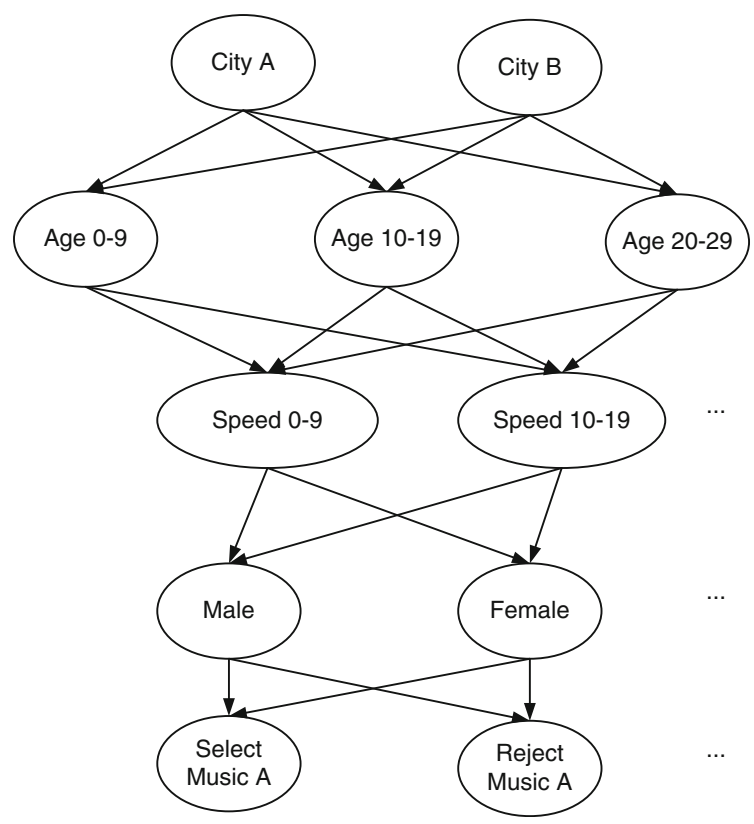

Fig. 12 Sample Bayesian networks for determining music preference and choice 
Table 2 Information about individuals who participated in the experiment

\begin{tabular}{lllllllll}
\hline & Individual 1 & Individual 2 & Individual 3 & Individual 4 & Individual 5 & Individual 6 & Individual 7 & Individual 8 \\
\hline Gender & Male & Female & Male & Male & Female & Female & Male & Male \\
Age & 29 & 30 & 27 & 26 & 25 & 25 & 35 & 30 \\
Height $(\mathrm{cm})$ & 170 & 170 & 170.8 & 167.7 & 158 & 164 & 177 & 180 \\
Weight $(\mathrm{kg})$ & 62 & 58 & 62 & 79.3 & 50 & 50 & 71 & 80 \\
Activity & Medium & Low & Low & High & Medium & Low & Low & Medium \\
Disease & None & None & None & None & None & None & None & None \\
\hline
\end{tabular}

recommends MusicA to the user. As the threshold value is getting larger, the recommended music can be more adequate for the exercise circumstance and satisfied by the user.

$\operatorname{Pr}($ CityA, Age10 - 19, Speed0 - 9, Male|SelectMusicA)

$=\frac{\operatorname{Pr}(\text { CityA }) \times \operatorname{Pr}(\text { Age 10-19|CityA }) \times \operatorname{Pr}(\text { Speed0 }-9 \mid \text { Age } 10-19) \times \operatorname{PR}(\text { Male } \mid \text { Speed0 }-9 \times \operatorname{Pr}(\text { SelectMusicA } \mid \text { Male }))}{\operatorname{Pr}(\text { SelectMusicA })}$

$\geq$ Threshold

\section{Experimental results}

\subsection{Experimental setup}

As shown in Table 2, eight different individuals participated in the experiment to test the customized game-like wireless interval training guidance system. All of these individuals are between the ages of 25 and 35, and three of the participants are female. Those individuals were randomly selected regardless of physical conditions, gender, or age.

Individual \#1 to Individual \#7 in Table 2 was requested to annotate their preference in music by using the music annotation user interface. Members in each individual group share gender, age, and residential area. Each song in the web database was annotated more than seven times.

With a modified version of the already existing Tabata Protocol interval training method (Fig. 1), we collected
10 sets of data with several attributes that may affect an individual's heart rate and exercise potential. While performing Tabata Protocol, a participant should step every 0.5 or $0.75 \mathrm{~s}$ during active periods on a $25 \mathrm{~cm}$-tall stool. For rest periods, we assumed the participants took a full rest. Each 22 min exercise session data set includes 13,219 attributes as shown in Table 3. Each Tabata Protocol session consists of three exercise periods and three rest periods.

Before starting an exercise session, other attributes such as age, height, and weight were recorded in the data base. During the $22 \mathrm{~min}$ session, the user's heart rate was monitored and recorded. Additionally, we recorded the inaccuracy of exercises after finishing our workout session. In our data, the inaccuracy results from the difference between the scheduled amount of exercise and the actual amount of exercise performed. As mentioned, the speed or
Table 3 13,219 attributes in one data set

\begin{tabular}{llll}
\hline Attributes & Value & Attributes & Value \\
\hline Gender & $\{$ male, female $\}$ & Days after period & Numeric \\
Age & Numeric & Number of exercises done today & Numeric \\
Height $(\mathrm{cm})$ & Numeric & Time & Numeric \\
Weight $(\mathrm{kg})$ & Numeric & Inaccuracy of exercise (\%) & Numeric \\
Activity level & $\{$ low, medium, high $\}$ & Time constant & Numeric \\
Disease name & string & Number of exercise period & Numeric \\
Hours of sleep & Numeric & Number of rest period & Numeric \\
Hours after a meal & Numeric & $\ldots$ & $\ldots$ \\
Medicine & String & 13,200 Heart rate variables & numeric \\
Hours after taking a medicine & Numeric & $($ every $0.1 \mathrm{~s})$ & \\
\hline
\end{tabular}




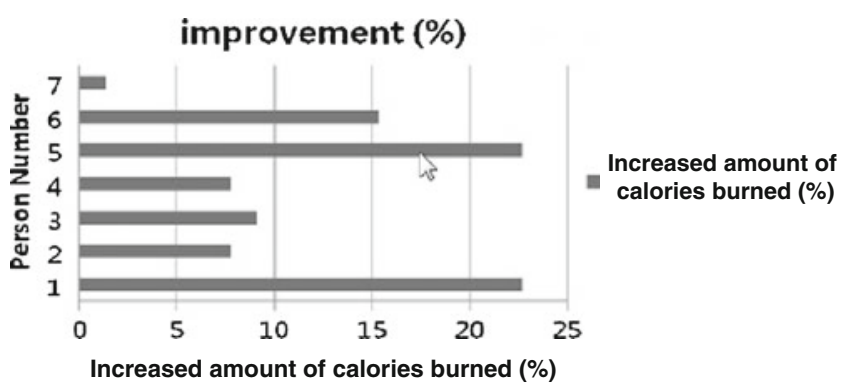

Fig. 13 The result of the exercise satisfying the conditions of the decision tree. This shows when the person follows conditions determined by $\mathrm{J} 48$ tree. The increase in the amount of calories burned is from $1.36 \%$ to $22.73 \%$

movement in the actual exercise can be obtained by using the 3-axis accelerometer on the iPhone.

With obtained data sets, the knowledge discovery and data mining techniques provides the extraction and classification of information that we need. A Java version C4.8 (J48) decision tree was generated by Weka (a data mining tool) using data along with data mining techniques. The J48 Decision tree classifier follows the following simple algorithm. First, a decision tree based on the attribute values of the available training data is created in order to classify a new item. Thus, whenever it encounters a training set it identifies the attribute that discriminates the various instances most clearly. Among the possible values of this feature, if there is any value for which there is no ambiguity, that is, for which the data instances falling within its category have the same value for the target variable, then it terminates that branch and assigns the target value that we obtained. For the other cases, it looks for another attribute that gives the highest information gain. Hence it continues in this manner until a clear decision of what combination of attributes gives a particular target value, or it runs out of attributes. In the event that it runs out of attributes, or if it cannot get an unambiguous result from the available information, it assigns this branch a target value that the majority of the items under this branch possess. In addition, if more data sets are accumulated in the database, the system adapts to achieve a more accurate classification. Conditions that extend exercise periods and shorten rest periods related to the time constants were obtained from the constructed $\mathrm{J} 48$ decision tree. For example, if the user has slept for more than $6 \mathrm{~h}$ the previous night or has eaten within

Table 4 Uncontrolled exercise protocol for ten data in phase 1

\begin{tabular}{lll}
\hline & Warm up & $\begin{array}{c}\text { Stepping on the stair per } 0.75 \mathrm{~s} \text { for } \\
5 \mathrm{~min}\end{array}$ \\
Repeat 3 times & 6 set exercises & $\begin{array}{c}6 \times \text { (stepping on the stair per } 0.5 \mathrm{~s} \\
\text { for } 20+10 \mathrm{~s} \text { rest) }\end{array}$ \\
& $\begin{array}{l}1 \mathrm{~min} \text { rest } \\
\text { Rest }\end{array}$ \\
Cool down & $\begin{array}{c}\text { Stepping on the stair per } 0.75 \mathrm{~s} \text { for } \\
5 \text { min }\end{array}$
\end{tabular}

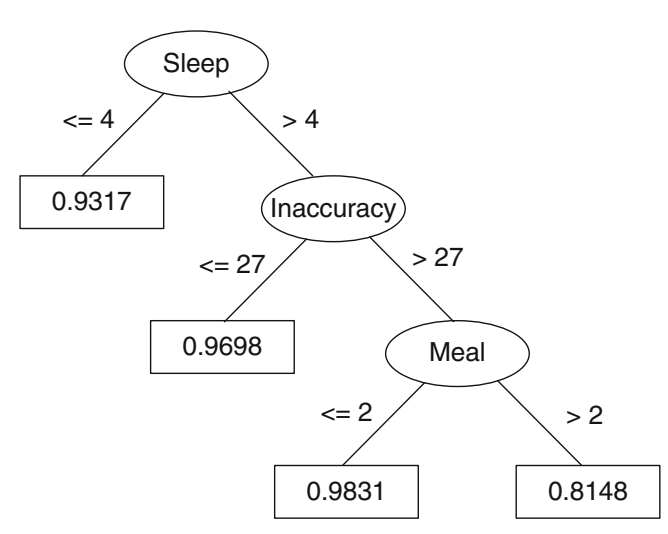

Fig. 14 The J48 decision tree shows conditions that make the time constant of four rising curves that have a characteristic of exponential sine hyperbolic curves maximized. The J48 decision tree that makes the time constant of three falling curves that have a characteristic of exponential curves minimized is also same. Sleep: the amount of hours to take a sleep before exercising. Inaccuracy: the inaccuracy between the accelerometer data and scheduled data. Meal: the amount of hours after having a meal

the last $2 \mathrm{~h}$, the system can tell the participant to exercise more. Or in other words, the system can tell the user to exercise for a longer period of time in order to maximize the exercise within the 22 min duration.

Exercise information, such as the starting and ending heart rate value, was also obtained. People exercised until the maximal heart rate reached the optimal rising curve in the exercise period. They resumed exercising when the minimal heart rate reached the optimal falling curve during the rest period as determined by the decision tree. By combining conditions related to time constants and an increase in the amount of exercise, we increased the amount of exercise while reducing fatigue.

As data is accumulated in the data base, the decision tree obtained from phase 2 is updated. As mentioned, the decision tree will become more accurate as more data is accumulated in the database.

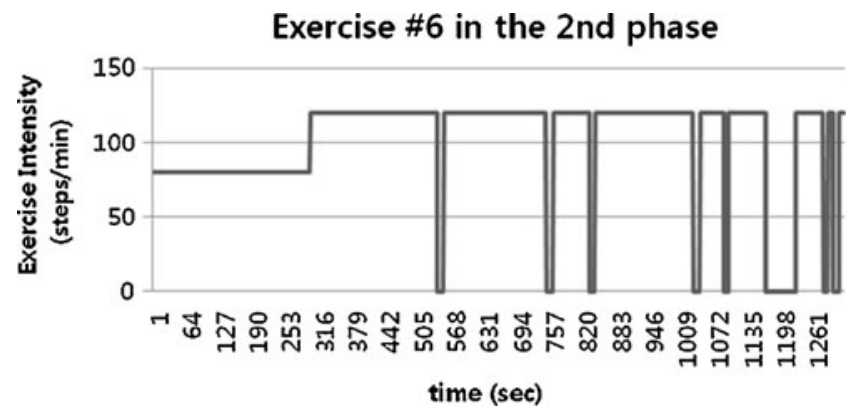

Fig. 15 The updated schedule of Tabata Protocol when following the constructed J48 decision tree. Intensity $120: 1$ step per 0.5 s. Intensity 75: 1 step per $0.75 \mathrm{~s}$ 


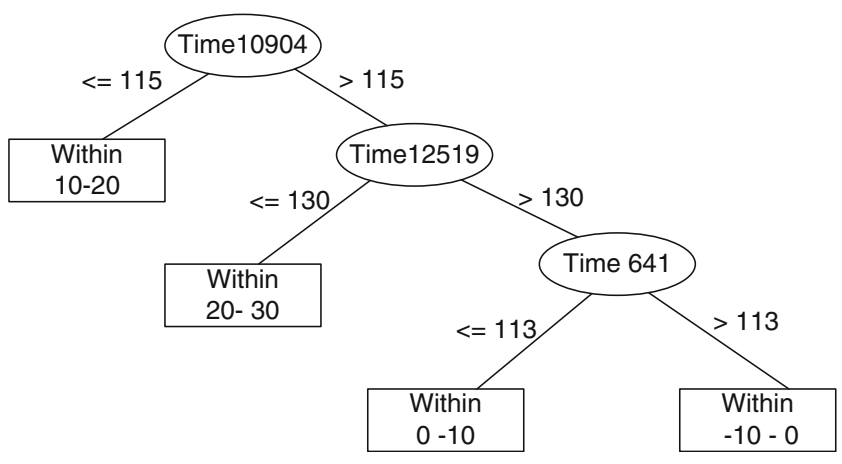

Fig. 16 J48 tree which shows conditions which make the amount of calories burned maximize. Within X-Y: X to Y percent increase of the amount of exercise. Time $\mathrm{X}$ : Heart rate at $\mathrm{X}$ time unit. (Each time unit: $0.1 \mathrm{~s}$ )

\subsection{Protocol results}

Seven different individuals (Individual \#1 to \#7) in Table 2 participated in five exercises that used the modified Tabata Protocol (Warm up-stepping on a platform every $0.75 \mathrm{~s}$ for $2 \mathrm{~min} 30 \mathrm{~s}$, six times of stepping on a platform every 0.5 for 20 and $10 \mathrm{~s}$ rest, $1 \mathrm{~min}$ rest). Since studies have shown that an individual's heart rate will return to a resting rate after $3 \mathrm{~min}$, individuals were asked to take a 3 min break between the five different exercise sessions. Afterwards, they completed the exercise satisfying the conditions of the decision tree. We observed an improvement of up to $22.73 \%$ in the amount of calories burned within $5 \mathrm{~min}$ and $30 \mathrm{~s}$. This proves our methods help people to exercise more within a given heart rate threshold range (Fig. 13).

Individual \#6 in Table 2 participated in the original 22 min modified Tabata interval training (Table 4). She finished ten sets of $22 \mathrm{~min}$ Tabata exercise sessions with different conditions on different days. Information related to one's conditions in Table 3 is stored and used to generate the J48 tree after completing ten interval trainings. The generated J48 tree after finishing ten Tabata interval trainings is shown below. This gives the conditions that maximize the time of exercise periods and minimize the time of rest periods. Conditions mentioned in Fig. 14 are updated and modified as data is accumulated.

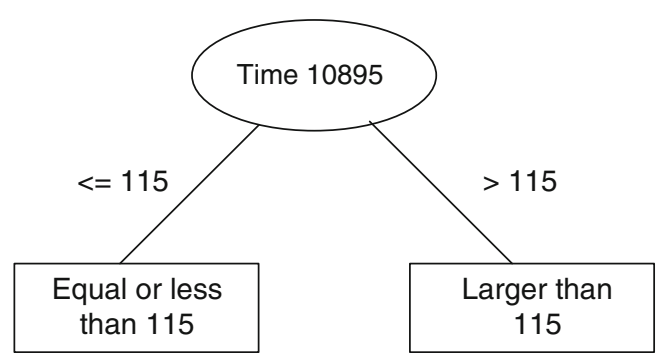

Fig. $17 \mathrm{~J} 48$ tree which shows conditions which make heart rate at $1089.5 \mathrm{~s}$ more than 115

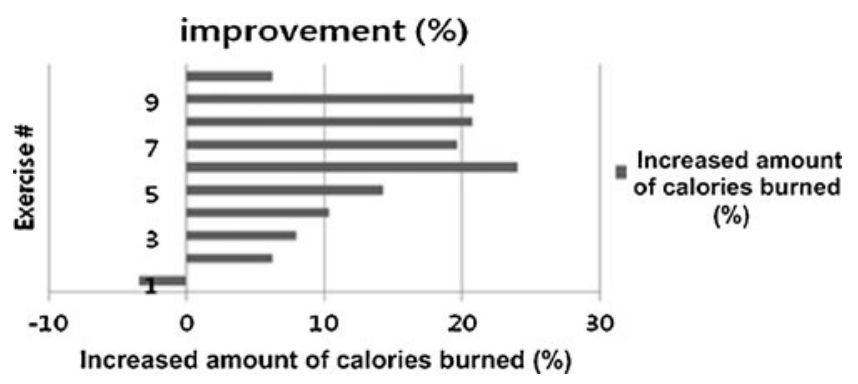

Fig. 18 The percentage of improvement in the amount of calories burned within $22 \mathrm{~min}$

The original schedule of the modified Tabata interval training is shown in Fig. 1. The participant follows conditions in Fig. 14 and exercises until the current heart rate reaches the level in the rising curve that has the maximum time constant among the individual's previous rising curves. The individual resumed exercising when the heart rate recovered to the lowest level of the heart rate curve with the minimum time constant. The changed schedule of exercise is shown in Fig. 15. We observed that the rest period of the 2 nd phase exercise is sparser and shorter than the original schedule.

After finishing the second ten sets of the Tabata interval training that fulfills conditions in Fig. 14, a new J48 tree was generated with 20 accumulated interval training data. The conditions that maximize the amount of the calorie expenditure are shown in Fig. 16. Other decision trees that satisfy conditions in Fig. 17 are obtained by backtracking. ten sets of Tabata interval training done in the second phase show an increase in the amount of calories burned by $24.09 \%$ (Fig. 18).

The results of the three sets of $22 \mathrm{~min}$ exercise data satisfying the J48 decision trees in Figs. 14 and 17 show more improvement in the amount of exercise. We see an improvement of up to $29.54 \%$ compared with the results of the original protocol (Fig. 19).

In the developed system, commands are given as a colored bar, sound, and vibration. The colored bar changes its color each time the person should step. For sound and vibration, the developed system gives either a short sound or vibration. Also, a message is displayed if the user doesn't

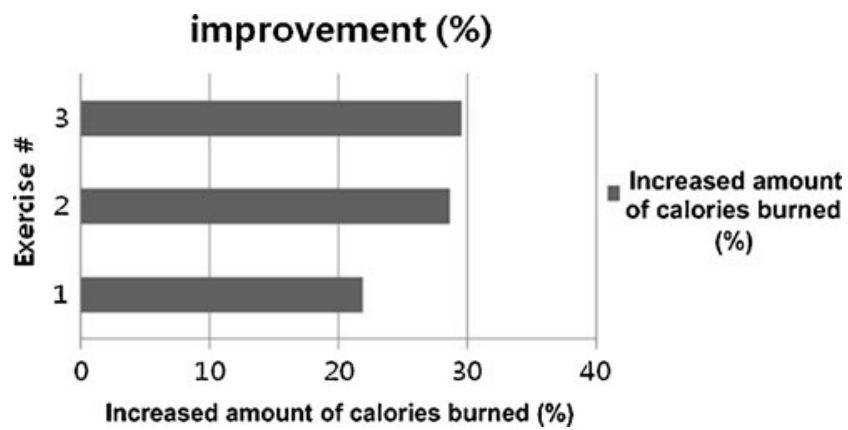

Fig. 19 The percentage of increase in the amount of calories burned within 22 min satisfying two J48 trees in Figs. 14 and 18 
Fig. 20 The accuracy of the exercise with given commands

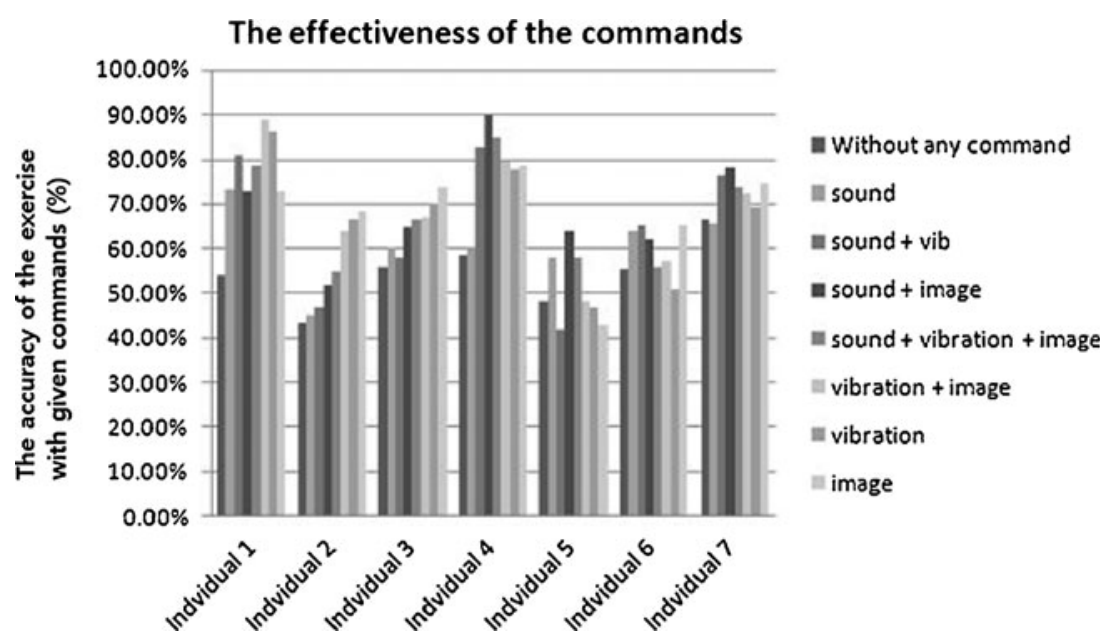

follow the given command (such as 'run faster' or 'run slower').Compared with the uncontrolled condition, the experiment (Fig. 20) shows that exercise commands generally help users to exercise more accurately. Especially for individual \#4, the accuracy of the exercise was improved from $53.97 \%$ to $88.71 \%$.

Figure 20 shows that the most effective command combination is different from each person. Hale [47] also mentions that different people require different time to perform information processing tasks depending on the type of information. This result also can be affected by the circumstance of the experiment. For example, the sound command is not a proper exercise command to the user in the noisy area or for the user who has a hearing problem.

Since the vibration affects the reading of the 3 -axis accelerometer data, it has more than $85 \%$ of the false positive rate (Fig. 21). When using probabilistic model (Fig. 22) to calculated true positive rate of the movement detection with using vibration commands, the true positive detection rate of Individual \#5 is 0.44 .

Figure 23 shows the amount of time to consume $80 \%$ of an iPhone's battery life. Utilizing images solely to give commands indicating exercise speed consumes the least energy compared with other command types and their combinations. In contrast, using sound commands require the most energy and interrupts users listening to music. As discussed, vibration causes high false positive rates of

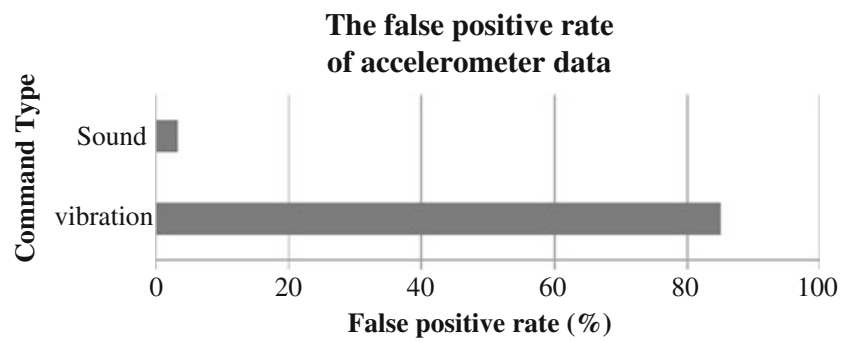

Fig. 21 The false positive rate of accelerometer data with and without vibration command accelerometer data and also consumes higher energy than image.

For \#6 in Table 2, the amount of time required to reach the same heart rate (137) increases as we repeat the interval training protocol (Fig. 24). This means Individual \#6 has adapted to the Tabata interval training protocol, and as a result, increases his endurance to complete the exercise and benefits the effect of cardiovascular build-up. As many researchers commented, this kind of high interval training helps the promotion of build-up in the cardiovascular system [48, 49]. Several studies of exercise training younger and older patients with heart failure have shown improvements in symptoms and exercise capacity and in many patho-physiological aspects of heart failure. This includes skeletal myopathy, ergoreceptor function, heart rate variability, endothelial function, and cytokine expression [50]. Compared with studies done before, which usually show improvement within 10 weeks.etc., signs of improvement in the amount of calories burned appeared within 2 weeks, since the decision tree and the interval training that originally helps cardiovascular build-up were combined together.

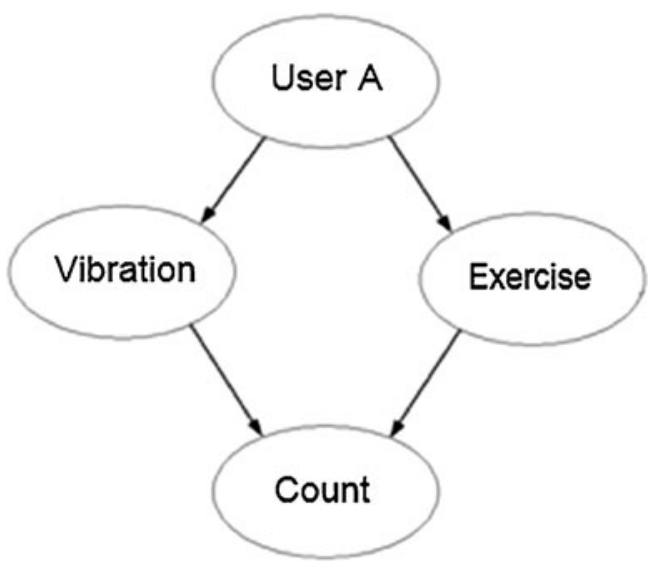

Fig. 22 Probabilistic model to calculate true positive rates using vibration command 


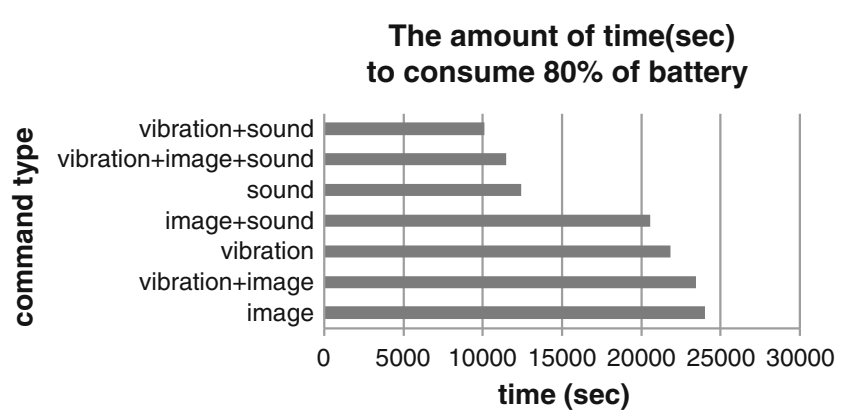

Fig. 23 The amount of required time to consume $80 \%$ of battery life

\subsection{Music recommendation results}

Figure 25 shows the number of refused songs among ten recommendations for Individual \#8. Compared with the method that recommends music preferred by people who share the same age range, gender, similar exercise intensity and residential area, Bayesian networks-based recommendation method shows better results for selecting suitable exercise music while interval training.

In addition, by using content-based filtering, if there is music that only a small number of individuals do not like, this song will not be recommended for that user, but still will be recommended to others until the rating is below the threshold level. Music is also selected by the intensity of the interval training. The appropriateness of music in the exercise context is more suitable, when considering the exercise context, the user's history, and the group that the user is included, than in the uncontrolled condition using random music. This contentbased, context-aware, and collaborative music recommendation system will choose suitable music for the user who is engaged in the interval training.

In addition, six out of the eight participants answered that the developed system positively affected motivation to participate in interval training due to the combination of music recommendations and social network systems. Since the music is chosen based on several aspects, including the user's age, exercise speed and gender, users thought the

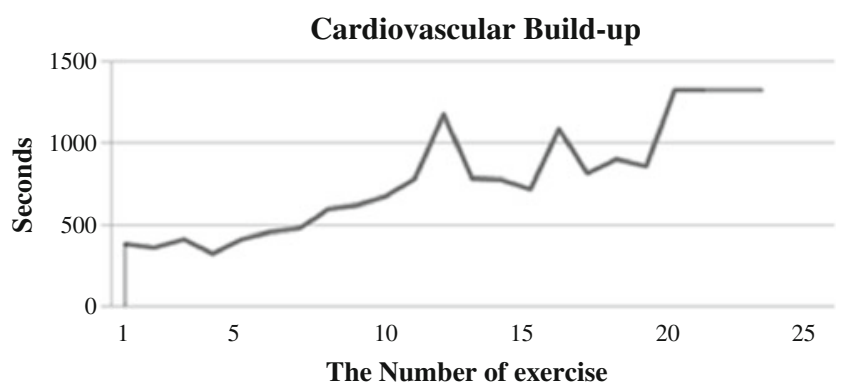

Fig. 24 The required time to reach a heart rate of 137. This shows that for the first exercise period in the $22 \mathrm{~min}$ Tabata protocol with the same heart rate threshold increased due to the adaptation and cardiovascular build-up

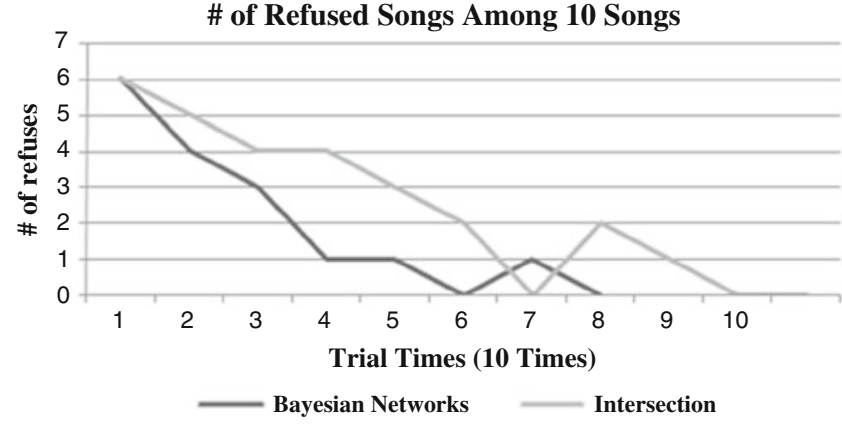

Fig. 25 The number of refused music among ten recommended

system provides long-term motivation to continue exercising while listening to music.

\section{Conclusion}

Interval training involves a series of intensive exercises coupled with recovery periods (or periods of low intensity activity). Despite interval training's health benefits, designing optimal interval training protocols and following given interval training protocols is not simple.

Our game-like social networking application with a music recommendation and exercise scheduling system on a light and small smart-phone is used to prompt participation in the interval training without any large or expensive equipment. The performance of the user can be measured by sensor readings, specifically accelerometers embedded within an iPhone. Our experiments show that customized interval training schedules and commands generated based on the user information increase the accuracy of the interval training up to $88.71 \%$.

A content-based, context-aware, and Bayesian networkbased collaborative filtering algorithm incorporates user music preferences and the exercise speed to play music to enhance performance. Based on user information combined with the information of other individuals in the same group and the speed of the interval training, the list of recommended music is generated and evolved. The experiments show that our Bayesian networks-based method with three recommendation techniques is better for recommending exercise music than the intersection methods. As more data related to the music is accumulated, the experiments show improvement in the selection of recommended music.

Computer-based analysis and classification, such as data mining, can be very helpful for developing optimized interval training protocols individually tailored for users. In this paper, a J48 tree in Weka was used to formulate the conditions that increase the amount of high-intensity activity within the 22 min program compared with original exercise program. Based on this information, we obtained up to $29.54 \%$ increase in the amount of calories burned compared with the original Tabata interval training protocol. 
Acknowledgement This research was supported by NLM (National Library of Medicine).

I would like to thank Alfred Heu and Kyujoong Lee for their help in performing the provided experiments, and to Professor William Kaiser for his helpful advice.

\section{References}

1. Billat LV (2001) Interval training for performance: a scientific and empirical practice: special recommendations for middle-and longdistance running. Part I: aerobic interval training. Sports Med 31 (1):13-31

2. Gorostiaga EM (1991) Uniqueness of interval and continuous training at the same maintained exercise intensity. Eur J Appl Physiol 63(2):101-107

3. Essen B (1977) Utilization of blood-borne and intramuscular substrates during continuous and intermittent exercise in man. $\mathbf{J}$ Physiol 265(2):489-506

4. Fox EL (1975) Frequency and duration of interval training programs and changes in aerobic power. J Appl Physiol Respir Environ Exerc Physiol 38(3):481-484

5. Dimeo FC (1999) Effects of physical activity on the fatigue and psychologic status of cancer patients during chemotherapy. Cancer 85(10):2273-2277

6. Tabata I (1996) Effects of moderate-intensity endurance and highintensity intermittent training on anaerobic capacity and [spacing dot above] VO2max. Med Sci Sports Exerc 28(10):1327-1330

7. http://itunes.apple.com/app/itgo-interval-trainer-go-the/

8. http://itunes.apple.com/app/iworkout-muse/

9. Tapia, E. M. (2007). Real-time recognition of physical activities and their intensities using wireless accelerometers and a heart rate monitor. The 11th International Conference on Wearable Computers.

10. Terry, P. C. (2006). Psychophysical effects of music in sport and exercise: an update on theory, research and application, Joint Conference of the Australian Psychological Society and the New Zealand Psychological Society.

11. Karageorghis, C. I. (2001). The magic of music in movement. Sport and Medicine Today.

12. Kleijnen, M. (2003). Factors influencing the adoption of mobile gaming services. In Mobile Commerce: technology, theory, and applications. 202-217.

13. Cano, P. (2005). Content-based music audio recommendation. Proc. ACM Multimedia, pp. 212-212.

14. http://www.apple.com/itunes/

15. Raymond, J. M. (2000) Content-based book recommending using learning for text categorization. In Proceedings of the Fifth ACM Conference on Digital Libraries.

16. Kwon OB (2003) "I know what you need to buy": context-aware multimedia-based recommendation system. Expert Syst Appl 25 (3):387-400

17. Van Setten, M. (2004). Context-aware recommendations in the mobile tourist application COMPASS. Lecture notes in computer science, 235.

18. Park H (2006) A context-aware music recommendation system using fuzzy bayesian networks with utility theory. Lect Notes Comput Sci 4223:970-979

19. Yang WS, Cheng HC, Dia JB (2008) A location-aware recommender system for mobile shopping environments. Expert Syst Appl 34(1):437-445

20. Kilpatrick M (2005) College students' motivation for physical activity: Differentiating men's and women's motives for sport participation and exercise. J Am Coll Health 54(2):87-94
21. Williams $P$ (1997) Effects of group exercise on cognitive functioning and mood in older women. Aust N Z J Public Health 21:45-52

22. Quinlan JR (1993) C4.5: Programs for machine learning. Morgan Kaufmann, San Mateo

23. Sarker S (2003) Understanding mobile handheld device use and adoption. Comm ACM 46(12):35-40

24. Knopf, R. C. (1983). Recreational needs and behavior in natural settings. Human behavior and environment: behavior and the natural environment. Vol. 6.

25. Knopf, R. C. (1987). Human behavior, cognition, and affect in the natural environment. Handbook of environmental psychology Vol. 1.

26. Kaplan, S. (1989). The experience of nature: A psychological perspective.

27. Hasan, M. (2008). The effects of music on the perceived exertion rate and performance of trained and untrained individuals during progressive exercise. Physical Education and Sport.

28. Szabo A (1999) The effects of slow- and fast-rhythm classical music on progressive cycling to voluntary physical exhaustion. J Sports Med 39(3):220-225

29. Wijnalda, G. (2005). A personalized music system for motivation in sport performance. IEEE pervasive computing.

30. http://www.facebook.com/

31. http://twitter.com/

32. Cole CR (1999) Heart-rate recovery immediately after exercise as a predictor of mortality. N Engl J Med 341(18):1351-1357

33. Mizuo J (2000) Exponential Hyperbolic Sine Function Fitting of Heart Rate Response to Constant Load Exercise. Jpn J Physiol 50 (4):405-412

34. Borg G (1987) Perceived exertion related to heart rate and blood lactate during arm and leg exercise. Eur J Appl Physiol 56(6):679-685

35. Janssen, P. G. J. M. (2001). Lactate threshold training. Human Kinetics.

36. van Ravenswaaij-Arts C (1993) Heart rate variability. Ann Intern Med 118(6):436-447

37. Lund-Johansen P (1999) Blood pressure and heart rate responses during physical stress in hypertension: modifications by drug treatment. Eur Heart J 1(B):10-17

38. Tuininga YS (1994) Heart rate variability in left ventricular dysfunction and heart failure: effects and implications of drug treatment. British heart journal 72(6):509-513

39. Poole, D. C. (1985). Response of ventilatory and lactate thresholds to continuous and interval training. J Appl Physiol 58(4):1115-1121

40. Borg, G. (1987). Perceived exertion related to heart rate and blood lactate during arm and leg exercise. Eur J Appl Physiol 56(6):679-685

41. Prather, J. C. (1997). Medical data mining: knowledge discovery in a clinical data warehouse. AMIA Annual Fall Symposium 101-105.

42. LeBlanc A (1988) Tempo preferences of different age music listeners. J Res Music Educ 36(3):156-168

43. LeBlanc A (1996) Music style preferences of different age listeners. J Res Music Educ 44(1):49-59

44. Christenson, P. G. (1988) Genre and gender in the structure of music preferences. Comm Res 15(3):282-301

45. Furman W (1992) Age and sex differences in perceptions of networks of personal relationships. Child Dev 63(1):103-115

46. Johnstone J (1957) Youth and popular music: a study in the sociology of taste. Am J Sociol 62(6):563-568

47. Hale S (1994) Global processing-time coefficients characterize individual and group differences in cognitive speed. Psychol Sci 5 (6):384-389

48. Wisloff U (2007) Superior cardiovascular effect of aerobic interval training versus moderate continuous training in heart failure patients: a randomized study. Circulation 115(24):3086-3094

49. MacVicar MG (1989) Effects of aerobic interval training on cancer patients' functional capacity. Nurs Res 38(6):348-51

50. Witham MD (2003) Exercise training as a therapy for chronic heart failure: can older people benefit? J Am Geriatr Soc 51(5):6 